\title{
KONTINUIERLICHE MESSUNGEN VON ATMOSPHÄRISCHEN KONDENSATIONSKERNEN
}

\author{
von P. Ackermann $(*)$, M. Bider $\left(f^{*}\right)$ \& F. Verzár $(* * *)$
}

In den Jahren 1952-1954 wurden von uns kontinuierliche Registrierungen der atmosphärischen Kondensationskerne über lange Perioden durchgeführt. An vier verschiedenen Orten der Schweiz wurden mit dem automatischen Kondensationskernzähler alle 15 Minuten Zählungen vorgenommen. Solche ReihenRegistrierungen waren bisher [abgesehen von einer kurzen Periode mit dem AITkE NZähler $\left(^{1}\right)$ ] noch nicht gemacht worden. Es war deshalb nötig, vorerst Erfahrungen zu sammeln, ehe ausführliche Analysen der Beobachtungen durchgeführt werden können. Ueber diese Messungen an einzelnen Orten berichtet im Folgeden jeder von uns gesondert $\left({ }^{* * *}\right)$.

Dem Eidg. Fonds für Arbeitsbeschaffung danken wir für die Prof̂. F. Verzár gewährte Hilfe zum. Bau der automatischen Kondensationskernzähler, sowie dem Eidg. Fond für Klimaforschung, welcher die Arbeit mit diesen Apparaten durch eine Donation an denselben ermöglicht hat.

$\left({ }^{*}\right)$ Paud Ackermans, Aexologische Station in Payerne der Eidg. Meteorologischen Zentralanstalt, Payerne (Schweiz).

(**) Dr. MAX Bider, Astronomisch-Meteorologische Anstalt der Universität Basel, Basel-Binningen (Schweiz).

$\left({ }^{* * *}\right)$ Prof. Dr. F. VerzÁR, Direktor des Physiologischen Instituts der Universität Basel, Basel (Schweiz).

$\left({ }^{* * *}\right)$ Vorträge gehalten an einem "Symposium über Kondensationskernzäh. lungen" an der Tagung der Schweizerischen Naturforschenden Gesellschaft (Sektion für Geophysik, Meteorologie und Astronomie) in Altdorf am 27. September 1954.

(1) Bradbury N. E. \& Meunon H. J., Terr. Magn. \& Atm. Electr. 43;232 (1938). 